\title{
LA ERUPCIÓN DEL VOLCÁN DE LOS GIGANTES (LAUTARO) EN 1883. ALGUNAS CONSIDERACIONES
}

MATEO MARTINIC B. ${ }^{\text {a }}$

\section{RESUMEN}

La actividad histórica de un centro volcánico ubicado en la zona andina de la Patagonia austral (Campo de Hielo Patagónico Sur) identifica como cerro Lautaro (49 01'S/ $73^{\circ} 33^{\prime} \mathrm{O}$ ), recogida inicialmente de la tradición indígena (aónikenk), ha sido una materia de preocupación para historiadores, exploradores y vulcanólogos en procura de su confirmación para la ciencia geográfica, hecho logrado recién en 1960. Sin embargo el historial de búsqueda y registro no contaba con la evidencia descriptiva de una erupción de la que ha venido a disponerse por una reciente publicación referida a las memorias del inglés William $\mathrm{H}$. Greenwood, a propósito de la cual se hacen algunas reflexiones y sugerencias.

PALABRAS CLAVE: Patagonia, Volcanismo.

\section{THE ERUPTION OF THE GIANT'S VOLCANO (LAUTARO) IN 1883. SELECTED CONSIDERATIONS}

\begin{abstract}
The historic activity of a volcanic center located at the Andean zone of Southernmost Patagonia (Southern Patagonia Ice Field) is identified as the Lautaro mountain $\left(49^{\circ} 01^{\prime} \mathrm{S} / 73^{\circ}\right.$ $33^{\top}$ ). First recorded from the indigenous tradition (Aónikenk), this has been a matter of concern for historians, explorers and volcanologist that wanted confirmation for the geographic sciences, fact that was achieved in 1960. Nevertheless the research history and records did not count with descriptive evidence of an eruption and this has changed since the recent publication of the memories of the british William H. Greenwood, which allows us to make some comments and suggestions.
\end{abstract}

KEY WORDS: Patagonia, vulcanism.

a Profesor Titular y Emérito, Centro de Estudios del Hombre Austral. Instituto de la Patagonia, Universidad de Magallanes. mateo.martinic@umag.cl 
La reciente publicación de las memorias del antiguo baqueano William $\mathrm{H}$. Greenwood (Patagonia Bravía, Grace \& Campbell Eds., 2015) en su variado $y$ ameno contenido incluye un a información de valor científico excepcional como es la referida a la descripción de una erupción volcánica, fenómeno natural de rara ocurrencia en la Patagonia, por tratarse no solo de un testimonio indubitable en cuanto experiencia de campo, sino también por ser el primer y hasta ahora único registro que permite atribuir su origen al hoy bien conocido volcán Lautaro, cuya actividad y ubicación precisa fueron por largo tiempo un misterio. Más importancia atribuimos al testimonio por haber sido obtenido en una época, 1883, en que la Patagonia era un territorio en mucho todavía desconocido y virtualmente despoblado.

Para entonces, deber reiterarse, las noticias históricas disponibles sobre esa clase de fenómenos naturales en la parte austral del continente americano eran escasas e imprecisas, máxime en lo tocante a la ubicación de los centros eruptivos, y se basaban por lo común en datos recogidos por viajeros de boca de los indígenas. Solo a contar de 1879, con el registro testimonial y grafico hecho por oficiales de una nave de la Armada Británica atribuido a un volcán situado en la zona andina de la Patagonia hacia los $49^{\circ}$ de latitud sur se pudo adelantar en la aclaración de ese misterio geográfico. Tal condición de desconocimiento se mantuvo de hecho hasta 1961 cuando el geógrafo y explorador británico Eric Shipton consiguió hacer la identificación confirmación del cono eruptivo, desde entonces definitivamente reconocido como volcán Lautaro (Martinic, 2008). Las manifestaciones tangibles de la actividad volcánica se referían a restos cinéreos y de material piroclástico, visibles en diferentes sectores del plateau andino patagónico y a fumarolas del cono volcánico, pero no se disponía de un registro valido sobre un erupción como el conocido para otros centros ubicados más al norte en la cordillera de los Andes. De allí la importancia que atribuimos al relato de Greenwood, sobre el cual cabe abundar.

La observación de la erupción fue hecha y registrada por Greenwood en fecha indeterminada de comienzos de 1883 cuando con algunos compañeros se hallaba acampado en un sector de la cuenca superior del río Coyle próximo a la meseta de las Vizcachas que, aproximadamente, podría situarse con la coordenada $50^{\circ} 50$ 'S y $73^{\circ} 30^{\prime} 0$, fenómeno que el baqueano atribuyó al volcán de los Gigantes, precisando la ubicación del cono eruptivo en la cordillera de los Andes detrás y hacia el interior del monte Fitz Roy, el Chaltén de los indígenas aónikenk. Interesa transcribir su relato: La noche estaba extrañamente oscura y sombría; no obstante, a ratos se animaba con sonidos estruendosos que, a veces, duraban minutos y retumbaban como cañonazos. De vez en cuando, todo el horizonte norte se iluminaba con un resplandor sobrenatural, del que salian llamaradas que aparentemente llegaban a gran altura. No pasamos muy buena noche en medio de todos estos fenómenos extraños y la mañana siguiente no nos trajo consuelo. Porque había una nube espesa que cubría todo el campo y no se podía ver más allá de 5 metros. Además, toda la atmosfera estaba impregnada de una ceniza blanca fina, la que rápidamente cubrió todo el terreno, con un capa de como 4 centímetros.

La carpa estaba totalmente tapada por esta ceniza y teníamos que sacudirla a cada rato para impedir que el peso la hundiera.

$\mathrm{Se}$ nos hizo extremadamente difícil respirar, estábamos muertos de sed y teníamos un gusto amargo y desagradable en la boca a causa del aire de plomo y cobre que estábamos inhalando [Esa composición la determinó el medico Thomas A. Fenton de Punta Arenas posteriormente a base de una muestra de ceniza que le llevó Greenwood].

Los caballos habían desaparecido por completo y no podíamos ir a buscarlos. Cuando probamos el agua del lago, nos pareció amargo como la hiel; pero, por suerte, había un pequeño manantial protegido bajo la orilla y su agua brotaba deliciosamente dulce y fresca. Alli llegamos todos los trastos disponibles y los llenamos a la carpa (cubiertos cuidadosamente); enseguida, los tres nos cobijamos tan rápidamente como pudimos, cerrando todas las aperturas para que no entrara este aire mortal.

Por algunas horas, lo pasamos muy mal, casi sofocados. Hacia la mitad de la tarde, se levantó una fuerte brisa del sur y empujó las densas nueves de ceniza de vuelta hacia el norte. 
Nunca olvidaré el alivio que sentimos cuando pudimos salir de nuevo y respirar aire puro; subimos a la colina más cercana para ver lo que había. Los caballos habian desaparecido por completo -no es de asombrarse pobres bestiassin agua, ni pasto que pudieran comer, tenían que irse o morir.

Por detrás del Chalten, se levantaba una inmensa columna de denso humo negro; a largos intervalos salian de ella, llamas brillantes, acompañadas de fuertes ruidos. El profundo runrún, o sonido retumbante o palpitante (no sé cómo describirlo), era decididamente lo más impresionante que yo había escuchado en $\mathrm{mi}$ vida.

Esta columna de humo se elevaba aparentemente hasta una altura de unos cientos de metros; luego, se abría tomando la forma de un abanico inmenso, más parecido a un plumero (de plumas de avestruz), que a cualquier otra cosa que se me ocurra. Evidentemente en las alturas había una fuerte corriente que barría la masa de humo de vuelta a la tierra. Después, la dirección del viento cambió otra vez -desde el norte ahora- $y$, en pocos minutos, estábamos nuevamente envueltos en una densa nube de cenizas enfermaste. Pero, como habíamos sellado todos los resquicios de la carpa y cubierto las puertas con mantas, esta ceniza infernal no pudo entrar; así y todo, nuestras bocas estaban heridas y nuestras lenguas y labios resecos $y$ con ampollas; no parecían refrescarse ni al tomar agua y la sensación constante de molestia estomacal no ayudaba nada.

Esta situación duró como 7 días sin parar; si no hubiera sido por los cambios en la dirección del viento -que ocurría cada cierto tiempo y nos proporcionaba aire fresco- no hubiéramos sobrevivido.

El cuarto día de la erupción fue el peor de todos, pero pasado eso, el viento sur sopló más constantemente y con más fuerza, lo que nos proporcionaba, cada día, períodos más largos para respirar.

A la octava mañana, hubo una helada fuerte, el sol salió claro y brillante y no subió más humo desde el volcán. Si no hubiera sido por la extraordinaria apariencia gris del pasto $y$ las matas, y la acidez del agua, nadie se hubiera podido imaginar la tremenda convulsión de la naturaleza que acababa de ocurrir.

La descripción transcrita debe valorarse tanto por su veracidad como por tratarse de la única conocida para un fenómeno de la especie en la parte austral de América con semejante grado de detalles circunstanciales sobre el "volcán de los Gigantes", ahora Lautaro, hecha desde un lugar situado aproximadamente a unos 200 kilómetros al sudeste, en línea recta, del mismo, lo que hace comparable el fenómeno en cuanto a su magnitud con las relaciones obtenidas para otros sucesos del género históricamente recientes, calificados como espectaculares, casos de los volcanes Hudson (1991) y Chaitén (2008).

En esta consideración hay dos aspectos en los que cabe detenerse para la debida ponderación del testimonio de William H. Greenwood. Uno, que según releemos la descripción no deja de llamarnos la atención y que dice con la seguridad de su afirmación en cuanto que el volcán, esto es, el centro activo, está situado detrás y hacia el interior (noroeste) del monte Chaltén o Fitz Roy, en un tiempo en que sus contemporáneos indígenas y civilizados (exploradores tales como Francisco P. Moreno, Carlos M. Moyano y otros) invariablemente aceptaban que esta última montaña nombrada "era" el volcán misterioso de la Patagonia. ¿Cómo y de quién, cabe preguntarse, pudo obtener Greenwood semejante convicción, firme además por lo reiterada, como se comprueba por la referencia previa contenida en la página 151 de la obra citada? Sorprendentemente porque en la historia conocida de la búsqueda e identificación del volcán activo de que se trata la primera certidumbre se tuvo recién en 1933, vale decir medio siglo después de la experiencia de campo del inglés y su confirmación debió aguardar todavía casi tres décadas más hasta ser aceptada por la ciencia geográfica.

No menos curioso nos resulta lo del nombre asignado por Greenwood al volcán (de los Gigantes), denominado que procedió en más de un siglo a la presencia del aventurero en la Patagonia y de tan rara mención cartográfica que lleva a pensar que su empleo pudo ser el fruto consecuente de un conocimiento erudito sobre mapas antiguos. Si así no fue, entonces cabe preguntarse nuevamente acerca de cómo 
Greenwood pudo enterarse de, y usar después, un topónimo virtualmente desconocido en su época aun para gente con conocimientos en geografía histórica.

Por ello, averiguar sobre ambos aspectos permanece como una cuestión abierta a la espera de algún investigador cuyo diligencia, de resultan provechosa, o su suerte como también suele ocurrir, puedan brindar una respuesta aclaratoria satisfactoria para una materia que, como esta, nos ha ocupado personalmente de manera recurrente en nuestros trabajos historiográficos.

Se nos ocurre, además, un par de sugerencias pertinentes. Una, teniendo en cuenta las observaciones de actividad volcánica atribuibles al Lautaro hechas en la década de 1870 durante trayectos de naves por los canales patagónicos, la posibilidad de verificar con un registro en los libros de bitácora de los vapores que cubrían la ruta Europa-Pacífico sur vía estrecho de Magallanes en el primer trimestre de 1883, pertenecientes a empresas tales como la Compañía Inglesa de Vapores (P. S. N. C.), y las alemanas Kosmos y Hamburg-Amerika Linie, para la cual sería necesario una revisión en los archivos que pudieran conservarse de esas entidades en repositorios británicos o alemanes.
De tenerse éxito en la búsqueda podría disponerse así de una fuente complementaria del valioso relato de Greenwood. La otra sugerencia atañe a los arqueólogos que trabajan en yacimientos de la zona sudoriental de la Patagonia (Santa Cruz y Magallanes) y se refiere a la toma de muestras subsuperficiales de cenizas volcánicas con miras a un posible posterior estudio a cargo de un especialista calificado como es el Dr. Charles Stern para los efectos de una identificación de muestras que pudieran corresponder a la tipología propia de las cenizas de volcán Lautaro. De ese modo, también, podría conocerse la amplitud territorial que habría alcanzado un fenómeno de aparente gran magnitud $e$ intensidad como ha sido el descrito por William Greenwood.

Está visto, así, que en el antiguo tema del volcanismo histórico en el sur de Patagonia queda harto por ver y averiguar.

\section{BIBLIOGRAFÍA}

Greenwood, W. H. (2015). Patagonia Bravía. Naturaleza, vidas y aventuras. Memorias originales del baqueano... En G. Grace \& D. S. Campbell (Eds.), Santiago.

Martinic B., M. (2008). Registro histórico de antecedentes volcánicos y sísmicos en la Patagonia Austral y la Tierra del Fuego. Magallania, 36(2), 5-18. 